\title{
Treatment of early-stage follicular lymphoma: do as I say, not as I do
}

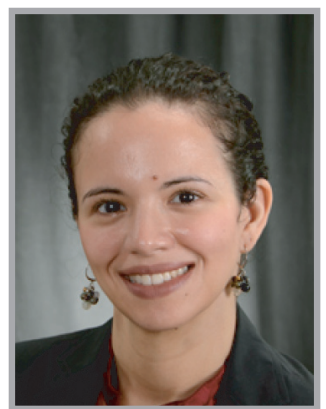

Carla Casulo ${ }^{1}$

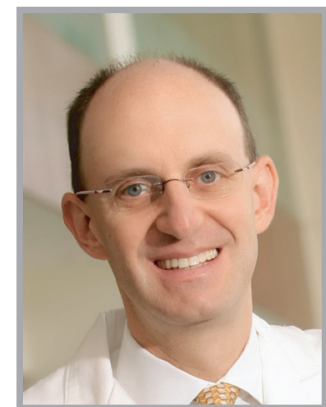

Jonathan W Friedberg*1
"With the exception of patients with stage I disease, there is no established standard of care for the treatment of follicular lymphoma, and therapeutic approaches

vary significantly worldwide."
Follicular lymphoma (FL) is the most common subtype of indolent non-Hodgkin's Lymphoma (NHL), and the second most common type of NHL in the USA and Europe, accounting for nearly one-quarter of all new cases of NHL [1]. With the exception of patients with stage I disease, there is no established standard of care for the treatment of FL, and therapeutic approaches vary significantly worldwide [2]. The National LymphoCare Study provides a rich resource for the study of FL. It is a multicenter, longitudinal, prospective registry of 2728 patients with newly diagnosed FL enrolled between 2004 and 2007 from over 200 different community and academic practice sites in the USA [3]. The first publication from the LymphoCare Study in 2009 described current practice patterns for patients with newly diagnosed FL and revealed that a wide variety of therapeutic strategies are being used across the USA. These included observation in $17.7 \%$ of patients, rituximab monotherapy in nearly $14 \%$, clinical trial referral in $6.1 \%$, radiation therapy in $5.6 \%$, chemotherapy only for $3.2 \%$ and chemotherapy plus rituximab in $51.9 \%$. Among the $17 \%$ of patients with stage I disease enrolled on this study, diverse treatment approaches were similarly observed, despite existing consensus guidelines recommending radiation therapy for patients with localized disease. Approximately onethird of patients were expectantly monitored, and another third received a rituximab-containing regimen (as either monotherapy or chemoimmunotherapy), but only approximately $25 \%$ of patients received standard radiotherapy. In a recent second analysis of the LymphoCare data to evaluate the outcomes of these early-stage patients, we found there were surprisingly no differences in overall survival (OS) between treatment groups, despite these varied treatment approaches. In fact, median OS was excellent, exceeding $80 \%$ at 5 years [4].

Recent data demonstrate that the OS of patients with FL is significantly longer than it was in previous years [5-7]. The LymphoCare study supports this, and suggests that for at least the small proportion of patients with localized FL, a hope for a prolonged disease remission can be sustained, and, most importantly, achieved through several means. The current literature guiding the management

'James P Wilmot Cancer Center, University of Rochester, 601 Elmwood Avenue, Box 704, Rochester, NY 14642, USA *Author for correspondence: jonathan_friedberg@urmc.rochester.edu

\section{"Recent data demonstrate that the overall survival of patients with follicular lymphoma is significantly longer than it was in previous years."}


"Given the excellent patient outcomes

observed across all treatment modalities, and in the absence of robust prospective evidence to uphold any one

therapeutic approach over another, the practicing oncologist is now challenged to maintain that radiation should remain the standard of care." of early-stage FL was established in the prerituximab era, and is largely based on several single-center retrospective studies. Despite limitations of patient selection and heterogeneous radiation doses and delivery, these observational series showed that patients with early-stage FL treated with radiotherapy have OS rates of up to $80 \%$ at approximately $7-8$ years, and similarly long relapse-free survival rates of up to $60 \%$ $[8,9]$. These studies provide the platform upon which both the European Society for Medical Oncology and the National Comprehensive Cancer Network recommend involved-field radiotherapy for patients with localized FL. Notwithstanding, the LymphoCare study found that only approximately $25 \%$ of patients received this treatment; rather, observation was the most common modality employed (30\%). Given the excellent patient outcomes observed across all treatment modalities, and in the absence of robust prospective evidence to uphold any one therapeutic approach over another, the practicing oncologist is now challenged to maintain that radiation should remain the standard of care. What should then be made of existing recommendations?

The LymphoCare report sheds important light on the implementation of evidence-based practice. Clinical practice guidelines, such as those published by the National Comprehensive Cancer Network, are developed in alliance with leaders in the oncology field in an effort to sustain consensus-driven management and ensure that patients receive diagnostic, therapeutic and preventive services that are most likely to yield optimal outcomes. Yet, the low number of clinicians following existing treatment recommendations for localized FL suggests there may be limitations to the successful execution of these guidelines. Perhaps the biggest impediment lies in a lack of appreciation on the management of early-stage FL, given its rarity. This, coupled with poor underlying data to support existing guideline adherence, may explain the observed lack of execution. These limitations are not unique to oncologists and, in fact, are common across many medical specialties [10]. In a recent publication analyzing practice guidelines for NHL in The Netherlands, Wennekes and colleagues found a need to improve implementation in many areas encompassing both diagnosis and treatment [11]. Suboptimal adherence has been attributed to patient characteristics, such as age and comorbidities, tumor characteristics and variations in geographic practice patterns $[11,12]$.
However, this may come at a price; one study evaluating quality of care in 1378 breast cancer patients found that better adherence to guidelines was related to superior OS and progression-free survival (PFS) [13,14]. In the LymphoCare study, differences in survival among patients with localized FL who underwent complete staging as dictated by practice guidelines were also noted [4]. Approximately $40 \%$ of patients underwent complete staging with bone marrow biopsies as well as diagnostic imaging with either a CT or $\left({ }^{18} \mathrm{~F}\right)$ fluorodeoxyglucose PET scan (referred to as 'rigorously staged' patients). Among these, rigorously staged patients had superior PFS compared with nonrigorously staged patients. While disease characteristics were similar between rigorous and nonrigorously staged patients, differences between these two groups of patients emerged across other categories. It appears that treatment selection may have been influenced by the degree of staging: more rigorously staged patients received chemoimmunotherapy or combined modality therapy (chemotherapy plus radiation) compared with nonrigorously staged patients $(\mathrm{p}<0.001)$. In this group, fewer rigorously staged patients were expectantly observed compared with nonrigorously staged patients. These findings underscore the relevance and importance of complete staging of all patients with FL to maximize outcomes.

The LymphoCare study highlights that despite diverse approaches in the treatment of stage I FL outside of evidence-based recommendations, most patients enjoyed excellent outcomes. The corollary to this is that patients who did not undergo complete staging with diagnostic imaging and bone marrow biopsy, as benchmarks dictate, had inferior PFS.

Guidelines are clearly meant to promote quality of care and clinicians' access to resources, and provide knowledge that can help guide decision-making in the management of cancer. Unfortunately, in the case of early-stage FL, our existing sources for those tools are outdated and potentially no longer relevant to the practicing clinician in today's era of chemoimmunotherapy and emerging targeted therapies. The LymphoCare registry provides a prospective, robust and up-todate source of data that we can capitalize on when making informed decisions on the management of rare cancers, such as early-stage FL. Large, randomized clinical trials in this setting are unlikely to happen largely because of cost and 
long-term follow-up that would be required for this favorable group of patients. However, the comparative effectiveness research provided by the LymphoCare study is a reflection of standard practice from which we can extrapolate to complement existing data. Focused efforts to encourage broad participation in these types of studies should continue in order to replenish and update the sources we use to provide optimum patient care.
Financial \& competing interests disclosure

$J W$ Friedberg is a Scholar in Clinical Research from the Leukemia and Lymphoma Society. JW Friedberg is on the advisory board for Genentech. The authors have no other relevant affiliations or financial involvement with any organization or entity with a financial interest in or financial conflict with the subject matter or materials discussed in the manuscript apart from those disclosed.

No writing assistance was utilized in the production of this manuscript.

\section{References}

Papers of special note have been highlighted as:

- of interest

-. of considerable interest

1 A clinical evaluation of the International Lymphoma Study Group classification of non-Hodgkin's lymphoma. The NonHodgkin's Lymphoma Classification Project. Blood 89(11), 3909-3918 (1997).

2 Zelenetz AD, Abramson JS, Advani RH et al. Non-Hodgkin's lymphomas. J. Natl Compr. Canc. Netw. 9(5), 484-560 (2011).

3 Friedberg JW, Taylor MD, Cerhan JR et al. Follicular lymphoma in the United States: first report of the national LymphoCare study. J. Clin. Oncol. 27(8), 1202-1208 (2009).

-. Multicenter, longitudinal, prospective registry of 2728 patients with newly diagnosed follicular lymphoma (FL) enrolled between 2004 and 2007 from over 200 different community and academic practice sites in the USA that describes current practice patterns for patients with newly diagnosed FL and reveals that a wide variety of therapeutic strategies are being used across the USA.
4 Friedberg JW, Byrtek M, Link BK et al. Effectiveness of first-line management strategies for stage I follicular lymphoma: analysis of the National LymphoCare Study. J. Clin. Oncol. 30(27), 3368-3375 (2012).

- This second published analysis from the LymphoCare study looks specifically at stage I FL.

5 Fisher RI, LeBlanc M, Press OW, Maloney DG, Unger JM. New treatment options have changed the survival of patients with follicular lymphoma. J. Clin. Oncol. 23(33), 8447-8452 (2005).

6 Liu Q, Fayad L, Cabanillas F, Hagemeister FB et al. Improvement of overall and failure-free survival in stage IV follicular lymphoma: 25 years of treatment experience at The University of Texas M.D. Anderson Cancer Center. J. Clin. Oncol. 24(10), 1582-1589 (2006).

7 Swenson WT, Wooldridge JE, Lynch CF, Forman-Hoffman VL, Chrischilles E, Link BK. Improved survival of follicular lymphoma patients in the United States. J. Clin. Oncol. 23(22), 5019-5026 (2005).

8 Guadagnolo BA, Li S, Neuberg D et al. Long-term outcome and mortality trends in early-stage, grade 1-2 follicular lymphoma treated with radiation therapy. Int. J. Radiat. Oncol. Biol. Phys. 64(3), 928-934 (2006).
9 Wilder RB, Jones D, Tucker SL et al. Longterm results with radiotherapy for stage I-II follicular lymphomas. Int. J. Radiat. Oncol. Biol. Phys. 51(5), 1219-1227 (2001).

10 Somerfield MR, Einhaus K, Hagerty KL, Brouwers MC, Seidenfeld J, Lyman GH; American Society of Clinical Oncology. American Society of Clinical Oncology clinical practice guidelines: opportunities and challenges. J. Clin. Oncol. 26(24), 4022-4026 (2008).

11 Wennekes L, Ottevanger PB, Raemaekers JM et al. Development and measurement of guideline-based indicators for patients with non-Hodgkin's lymphoma. J. Clin. Oncol. 29(11), 1436-1444 (2011).

12 Charlson M, Szatrowski TP, Peterson J, Gold J. Validation of a combined comorbidity index. J. Clin. Epidemiol. 47(11), 1245-1251 (1994).

13 Cheng SH, Wang CJ, Lin JL et al. Adherence to quality indicators and survival in patients with breast cancer. Med. Care 47(2), 217-225 (2009).

14 Yates JW, Chalmer B, McKegney FP. Evaluation of patients with advanced cancer using the Karnofsky performance status. Cancer 45(8), 2220-2224 (1980). 